\title{
Time Dependence of the Cosmological Parameters in the Framework of Brans-Dicke Theory
}

\author{
'Sudipto Roy, ${ }^{2}$ Avik Ghosh, ${ }^{3}$ Adrika Dasgupta \\ ${ }^{1}$ Department of Physics, St. Xavier's College, Kolkata, West Bengal, India \\ 2,3Post Graduate Student of Physics (2017-2019), St. Xavier's College, Kolkata, India \\ Emails: 1roy.sudipto@sxccal.edu, ${ }^{2}$ avikghosh98@gmail.com, ${ }^{3}$ adrika.dasgupta1995@gmail.com
}

\begin{abstract}
In the framework of Brans-Dicke (BD) theory of gravitation, the time dependence of some cosmological parameters have been determined in the present study, for an universe having a FRW space-time with zero spatial curvature. The time variations of the energy density, BD parameter, equation of state (EoS) parameter have been determined, from the field equations of the BD theory, in the initial part of this model. For this purpose, we have used ansatzes relating the scalar field with the scale factor and also linking the BD parameter with the scalar field. For these calculations, an empirical expression for the scale factor has been used. This scale factor has been so chosen that it leads to a signature flip of the deceleration parameter from positive to negative in the course of its evolution with time, indicating a change of phase from decelerated expansion to accelerated expansion. Time dependence of the density parameters for matter and dark energy has been studied here. Using their expressions we have determined the time dependence of the densities of matter and dark energy. The time variations of all these parameters have been shown graphically. Apart from them, we have also shown the variations of the deceleration parameter and the $\mathrm{BD}$ parameter as functions of the scalar field graphically.
\end{abstract}

Keywords : Cosmology, Dark Energy, Brans-Dicke Theory, Gravitational Constant, Cosmic Acceleration.

\section{INTRODUCTION}

It has been confirmed by recent astrophysical observations that the accelerated expansion of the universe is one of the most important and interesting phenomena in the field of cosmology [1-5]. A new form of energy, known as dark energy (DE), with a negative pressure, has been found to be responsible for the accelerated expansion of the universe. An extensive research is going on, throughout the world, to discover the true nature and dynamics of DE. To explain this accelerated expansion of the universe, following a decelerated expansion, various models have been proposed. A recent study, using the data from supernova, has clearly shown that there has been a signature flip of the deceleration parameter of the universe, from positive to negative, indicating a change of phase from deceleration to acceleration [6]. Astrophysical observations show that the present phase of accelerated expansion, which is a very recent phenomenon, was preceded by a phase of decelerated expansion [7]. It is important for the structure formation of the universe and its nucleosynthesis. According to different studies in this regard, beyond a certain redshift $(z)$ value the universe definitely had a phase of decelerated expansion $[2,8]$.

The present theoretical study is based on the BransDicke (BD) theory of gravitation. In 1961, this theory 
was developed by Robert H. Dicke and Carl H. Brans, based on an earlier work by Pascual Jordan in 1959 [9]. The Brans-Dicke theory (also known as the Jordan-Brans-Dicke theory) is a theoretical framework to explain gravitation, like the famous theory of general relativity proposed by Albert Einstein. It is known as a scalar-tensor theory, a theory of gravitation in which the gravitational interaction is mediated by a scalar field as well as the tensor field of general relativity. The gravitational constant $(G)$ is not a constant here. Its reciprocal, i.e. $1 / G$, is replaced by a scalar field $\phi$ which varies with time. BD theory is characterized by this $\phi$ and a dimensionless coupling parameter $(\omega)$ that determines the characteristics of space-time geometry. It is regarded as a natural extension of general theory of relativity whose results can be reproduced by $\mathrm{BD}$ theory in the limit of an infinite $\omega$ and a constant value of $\phi[10]$. BD theory has a very important place among the existing theories which are capable of explaining early and late time characteristics of the universe and it has solved the problems connected to the theory of inflation [11]. A generalized version of the BD theory was proposed, where $\omega$ was regarded as a function of the scalar field $\phi$ [12-14]. To explain the features of the expanding universe, in terms of cosmological parameters, various models have been developed on the basis of the BD theory $[10,15-18]$.

In the present article, BD field equations (for a flat, homogeneous and isotropic space-time) have been used to determine time dependence of the coupling parameter $(\omega)$, equation of state (EoS) parameter (denoted by $\gamma$ here) and energy density $(\rho)$. Time variation of the BD parameter $(\omega)$ was studied earlier by many groups, using scale factors that have power law dependence upon time $[10,17,18]$. Since the universe is known to have made a transition from decelerated expansion to accelerated expansion, the deceleration parameter $(q)$ must have a dependence upon time $[2,19]$. Keeping this fact in view, we have used an empirical scale factor (a) that generates a time dependent deceleration parameter which changes sign from a positive to a negative value. For the present calculations, we have assumed power law dependence of $\omega$ upon $\phi$ and also of $\phi$ upon $a$.

In the initial part of our theoretical model, we have determined the time dependence of the cosmological parameters such as the BD parameter, energy density, equation of state parameter for curvature constant $k=0$ i.e., for flat space. In the next part of the study, a simple cosmological model has been proposed, considering the non-conservation of the matter content of the universe. One is quite likely to assume an increase of dark energy with time at the cost of the other main constituent of the universe, i.e. matter, in the present matter dominated era, because, the dark energy is known to be responsible for the accelerated expansion, preceded by a phase of decelerated expansion of the universe. Present percentage of dark energy has been found to be approximately seventy and the percentage of matter (dark + baryonic) has been found to be approximately thirty [8]. In this part, an empirically chosen function $f(t)$ has been used, which increases with time from zero to unity. Expressions representing the time dependence of the relative proportions of matter and dark energy of the universe have been obtained from $f(t)$, considering the matter content to be decaying into dark energy. Using these expressions, the time dependence of the individual densities of matter and dark energy has been determined. The results of the present study, regarding the time variations of some cosmological parameters, are found to have sufficient consistency with the findings of some other recent studies (carried out in the framework of general relativity of Einstein), implying the theoretical correctness of this model. 


\section{METHOD OF FORMULATION}

\section{Brans-Dicke Field Equations}

The action, in the Brans-Dicke theory of gravitation, is expressed as,

$$
\mathcal{A}=\frac{1}{16 \pi} \int d^{4} x \sqrt{-g}\left(\phi R+\frac{\omega(\phi)}{\phi} g^{\mu v} \partial_{\mu} \phi \partial_{\nu} \phi+\mathcal{L}_{m}\right)
$$

In the above equation, $g$ denotes the determinant of the metric tensor $g^{\mu \nu}, \mathcal{L}_{m}$ denotes the Lagrangian for matter, $R$ stands for the Ricci scalar, $\phi$ denotes the Brans-Dicke scalar field and $\omega$ represents BransDicke parameter. This dimensionless parameter $(\omega)$ is regarded as a function of the scalar field $(\phi)$ in generalized BD theory.

One gets the following field equations by the variation of action $(\mathcal{A})$, given by equation $(1)$.

$$
\begin{aligned}
R_{\mu \nu}-\frac{1}{2} R g_{\mu \nu}= & \frac{1}{\phi} T_{\mu \nu}-\frac{\omega}{\phi^{2}}\left[\phi_{, \mu} \phi_{, v}-\frac{1}{2} g_{\mu \nu} \phi_{, \beta} \phi^{\prime \beta}\right] \\
& -\frac{1}{\phi}\left[\phi_{\mu ; \nu}-g_{\mu \nu}\left\{\frac{\partial^{2} \phi}{\partial t^{2}}-\nabla^{2} \phi\right\}\right] \\
& \frac{\partial^{2} \phi}{\partial t^{2}}-\nabla^{2} \phi=\frac{1}{2 \omega+3} T
\end{aligned}
$$

$R_{\mu \nu}$ denotes the Ricci tensor and $T_{\mu \nu}$ stands for the energy-momentum tensor. In equations (2) and (3), a semicolon denotes a covariant derivative and a comma represents an ordinary derivative with respect to $x^{\beta}$.

The energy-momentum tensor $\left(T_{\mu \nu}\right)$ for the cosmic constituents is expressed as,

$$
T_{\mu \nu}=(\rho+P) u_{\mu} u_{\nu}+g_{\mu \nu} P
$$

Here, $\rho$ is the energy density, $P$ denotes the isotropic pressure, $u_{v}$ denotes the four-velocity vector and $T$ stands for the trace of the tensor $T_{i j}$. This form of the energy-momentum tensor is based on an assumption that the total matter-energy content of the expanding universe behaves as a perfect fluid. In a co-moving coordinate system with $u^{v}=(0,0,0,1)$ we have $g_{\mu \nu} u^{\mu} u^{v}=1$.

In Friedmann-Robertson-Walker (FRW) space-time, the line element for a homogeneous and isotropic universe, is given by the following expression.

$d s^{2}=-d t^{2}+a^{2}(t)\left[\frac{d r^{2}}{1-k r^{2}}+r^{2} d \theta^{2}+r^{2} \sin ^{2} \theta d \xi^{2}\right]$

Where $a(t)$ is the scale factor, $t$ is cosmic time, $k$ denotes the spatial curvature. Here, $r, \theta$ and $\xi$ denote the three spherical polar coordinates. The curvature parameter $k$ describes the closed, flat and open universes corresponding to $k=1,0,-1$ respectively. For all calculations of the present study we have taken the spatial curvature $(k)$ to be zero.

Taking $k$ to be zero, equation (5) takes the following form.

$d s^{2}=-d t^{2}+a^{2}(t)\left[d r^{2}+r^{2} d \theta^{2}+r^{2} \sin ^{2} \theta d \xi^{2}\right]$

For a universe filled with a perfect fluid, the following three equations are the field equations of the BD theory, in the geometry of a homogeneous and isotropic space-time expressed by equation (5). These equations have been obtained by combining the expressions represented by equations (2), (3), (4) and (5).

$$
\begin{gathered}
3 \frac{\dot{a}^{2}+k}{a^{2}}+3 \frac{\dot{a} \dot{\phi}}{a \phi}-\frac{\omega \dot{\phi}^{2}}{2 \phi^{2}}=\frac{\rho}{\phi} \\
2 \frac{\ddot{a}}{a}+\frac{\dot{a}^{2}+k}{a^{2}}+\frac{\omega \dot{\phi}^{2}}{2 \phi^{2}}+2 \frac{\dot{a} \dot{\phi}}{a \phi}+\frac{\ddot{\phi}}{\phi}=-\frac{P}{\phi} \\
\frac{\ddot{\phi}}{\phi}+3 \frac{\dot{a} \dot{\phi}}{a \phi}=\frac{\rho-3 P}{2 \omega+3} \frac{1}{\phi}-\frac{\dot{\omega}}{2 \omega+3} \frac{\dot{\phi}}{\phi}
\end{gathered}
$$

In these field equations, $\gamma(\equiv P / \rho)$ denotes the equation of state (EoS) parameter for the cosmic fluid, 
which has been treated as a function of time in the present study.

\section{Theoretical Model}

\subsection{Determination of Expressions for $\rho, \gamma$ and $\omega$}

For the convenience of calculations, we have used the following symbols.

$a_{1}=\frac{\dot{a}}{a}, a_{2}=\frac{\ddot{a}}{a}, a_{3}=\frac{\dot{a}^{2}+k}{a^{2}}, \phi_{1}=\frac{\dot{\phi}}{\phi}$ and $\phi_{2}=\frac{\ddot{\phi}}{\phi}$

Incorporating the above symbols in equations (7), (8) and (9), we get,

$$
\begin{gathered}
3 a_{3}+3 a_{1} \phi_{1}-\frac{\omega}{2} \phi_{1}^{2}=\frac{\rho}{\phi} \\
2 a_{2}+a_{3}+\frac{\omega}{2} \phi_{1}^{2}+2 a_{1} \phi_{1}+\phi_{2}=-\frac{P}{\phi} \\
\phi_{2}+3 a_{1} \phi_{1}=\frac{\rho-3 P}{2 \omega+3} \frac{1}{\phi}-\frac{\dot{\omega} \phi_{1}}{2 \omega+3}
\end{gathered}
$$

From equations (10) and (11), $\frac{\rho-3 P}{\phi}$ has been calculated and substituted into equation (12) to obtain the following equation.

$$
\phi_{2}+3 a_{1} \phi_{1}=\frac{6 a_{3}+9 a_{1} \phi_{1}+\omega \phi_{1}^{2}+3 \phi_{2}+6 a_{2}}{2 \omega+3}-\frac{\dot{\omega} \phi_{1}}{2 \omega+3}
$$

In the present study we have used the following empirical relations for $\phi$ and $\omega$ respectively. These parameters have been assumed to have power-law relations with $a$ and $\phi$ respectively.

$$
\begin{aligned}
& \phi=\phi_{0}\left(\frac{a}{a_{0}}\right)^{l}=\phi_{0} a^{l} \\
& \omega=\omega_{0}\left(\frac{\phi}{\phi_{0}}\right)^{m}
\end{aligned}
$$

where, $a\left(t_{0}\right)=a_{0}=1$ and $\phi\left(t_{0}\right)=\phi_{0}$.

Here $l$ and $m$ are constant parameters. The symbols $\phi_{0}$ and $\omega_{0}$ denote the proportionality constants, representing the present values of the parameters $\phi$ and $\omega$ respectively.

The ansatz of $\phi$, given by equation (14), has been taken from some earlier studies in this regard $[15,19$, 20]. The logic behind choosing the above ansatz for $\omega$ is the fact that this is considered to be a function of the scalar field $(\phi)$ in the generalized version of Brans-Dicke theory $[13,14]$.

Calculating $\phi_{1}$ and $\phi_{2}$ from equation (14) and $\dot{\omega}$ from equation (15) we get,

$$
\begin{gathered}
\phi_{1}=l H \\
\phi_{2}=l(l-1) H^{2}-l q H^{2} \\
\dot{\omega}=\omega m l H
\end{gathered}
$$

Here we have used the standard expressions of the Hubble parameter $(H)$ and deceleration parameter $(q)$, which are $H=\frac{\dot{a}}{a}$ and $q=-\left(\frac{\ddot{a} a}{\dot{a}^{2}}\right)$ respectively.

Putting the values of all parameters for $t=t_{0}$ in equation (10), we have found the following expression for $\omega_{0}$.

$$
\omega_{0}=\frac{2}{\phi_{10}^{2}}\left(3 a_{30}+3 a_{10} \phi_{10}-\frac{\rho_{0}}{\phi_{0}}\right)
$$

Substituting $\dot{\omega}=\omega m l H$ in equation (13), we get the following expression for $m$ by using all parameter values for $t=t_{0}$.

$$
\begin{aligned}
m= & \frac{\left(6 a_{30}+9 a_{10} \phi_{10}+\omega_{0} \phi_{10}^{2}+3 \phi_{20}+6 a_{20}\right)}{\omega_{0} l H_{0} \phi_{10}} \\
& -\frac{\left(2 \omega_{0}+3\right)\left(\phi_{20}+3 a_{10} \phi_{10}\right)}{\omega_{0} l H_{0} \phi_{10}}
\end{aligned}
$$

Here $a_{10}=H_{0}, a_{20}=-q_{0} H_{0}^{2}, a_{30}=H_{0}^{2}, \phi_{10}=$ $l H_{0}, \phi_{20}=l(l-1) H_{0}^{2}-l q_{0} H_{0}^{2}$. 
Using equations (15), (19) and (20) one gets the following expression for $\omega$.

$$
\begin{gathered}
\omega=\frac{2}{\phi_{10}^{2}}\left(3 a_{30}+3 a_{10} \phi_{10}-\frac{\rho_{0}}{\phi_{0}}\right) \\
\times\left(\frac{\phi}{\phi_{0}}\right)^{\frac{\left(6 a_{30}+9 a_{10} \phi_{10}+\omega_{0} \phi_{10}^{2}+3 \phi_{20}+6 a_{20}\right)-\left(2 \omega_{0}+3\right)\left(\phi_{20}+3 a_{10} \phi_{10}\right)}{\omega_{0} l H_{0} \phi_{10}}}
\end{gathered}
$$

From equation (10), the following expression for $\rho$ is obtained.

$$
\rho=\phi\left(3 a_{3}+3 a_{1} \phi_{1}-\frac{\omega}{2} \phi_{1}^{2}\right)
$$

Dividing equation (11) by (10), one gets the following expression for the EoS parameter $(\gamma)$.

$$
\gamma=-\left(\frac{2 a_{2}+a_{3}+\frac{\omega}{2} \phi_{1}^{2}+2 a_{1} \phi_{1}+\phi_{2}}{3 a_{3}+3 a_{1} \phi_{1}-\frac{\omega}{2} \phi_{1}^{2}}\right)
$$

The time dependence of the quantities $a_{1}, a_{2}, a_{3}, \phi$, $\phi_{1}$ and $\phi_{2}$, used in the above expressions of $\omega, \rho$ and $\gamma$, can be obtained from the following discussion of the scale factor used in the present study.

\subsection{The Scale Factor}

The universe had a phase of decelerated expansion before the beginning of its present phase of accelerated expansion, as obtained from astrophysical observations [15, 21, 22]. The deceleration parameter (q) must have undergone a signature flip due to this change of phase. The scale factor, used for the present study, has a functional form that ensures a change of sign of the deceleration parameter from positive to negative. This function is a product of exponential function and power law, which was used by Roy et al. and Pradhan et al. [23, 24]. It is expressed as,

$$
a(t)=a_{0}\left(\frac{t}{t_{0}}\right)^{\alpha} e^{\beta\left(t-t_{0}\right)}
$$

In Equation (24), one must have $\alpha, \beta>0$ to ensure an increase of scale factor with time. The BD scalar field $(\phi)$, Hubble parameter $(H)$ and the deceleration parameter $(q)$, based on this scale factor, are expressed as,

$$
\begin{gathered}
\phi=\phi_{0}\left(\frac{a}{a_{0}}\right)^{l}=\phi_{0}\left(\frac{t}{t_{0}}\right)^{l \alpha} e^{l \beta\left(t-t_{0}\right)} \\
H=\frac{\dot{a}}{a}=\beta+\frac{\alpha}{t} \\
q=-\frac{\ddot{a} a}{\dot{a}^{2}}=\frac{\alpha}{(\alpha+\beta t)^{2}}-1
\end{gathered}
$$

For $0<\alpha<1$, one gets $q>0$ at $t=0$ and, in the limit of $t \rightarrow \infty$, one gets $q \rightarrow-1$. It shows a clear change of sign for the deceleration parameter with time. The following conditions have been used, in equations (26) and (27), to determine the values of the constant parameters $(\alpha, \beta)$.

\section{Condition 1:}

$H_{t=t_{0}}=H_{0}$ (as obtained from observations)

\section{Condition 2:}

$q_{t=t_{0}}=q_{0}$ (as obtained from observations)

Using equations (26), (27), (28) and (29), we get,

$$
\begin{gathered}
\alpha=H_{0}^{2} t_{0}^{2}\left(q_{0}+1\right)=4.76 \times 10^{-01} \\
\beta=H_{0}\left[1-H_{0} t_{0}\left(q_{0}+1\right)\right]=1.25 \times 10^{-18}
\end{gathered}
$$

Using equation (24), the redshift parameter $(z)$ can be expressed as,

$$
z=\frac{a_{0}}{a}-1=\left[\left(\frac{t}{t_{0}}\right)^{-\alpha} e^{-\beta\left(t-t_{0}\right)}-1\right]
$$

For the present study we have used the following values of cosmological parameters: 


$$
\begin{aligned}
& \begin{aligned}
H_{0} & =72 \mathrm{Kms}^{-1} \mathrm{Mpc}^{-1}=2.33 \times 10^{-18} \mathrm{~s}^{-1} \\
& =7.35 \times 10^{-11} \mathrm{yr}^{-1}
\end{aligned} \\
& \begin{aligned}
t_{0} & =14 \text { billion years }=4.415 \times 10^{17} \mathrm{~s} \\
\phi_{0} & =\frac{1}{\mathrm{G}_{0}}=1.498 \times 10^{10} \mathrm{~N}^{-1} \mathrm{Kg}^{2} \mathrm{~m}^{-2} \\
\rho_{0} & =9.9 \times 10^{-27} \mathrm{Kgm}^{-3} \\
q_{0} & =0.55
\end{aligned}
\end{aligned}
$$

Using this scale factor (eqn. 24), the characteristics of $\omega, \rho$ and $\gamma$ (based on eqns. 21, 22, 23) have been determined.

\subsection{Range of Values of the parameter $l$}

The parameter $l$ controls the time evolution of the scalar field $(\phi)$ as per equation (14). Time variations of $\omega, \rho$ and $\gamma$ depend on $l$, as we know that both $m$ and $\omega_{0}$ are functions of $l$ (as per eqns. 19, 20).

The following expression of the gravitational constant $(G)$, which is the reciprocal of the BD scalar field $(\phi)$, has been obtained from Equation (25).

$$
G=\frac{1}{\phi}=\frac{1}{\phi_{0}\left(\frac{a}{a_{0}}\right)^{l}}=\frac{1}{\phi_{0}}\left(\frac{t}{t_{0}}\right)^{-l \alpha} \exp \left[-l \beta\left(t-t_{0}\right)\right]
$$

According to some cosmological studies, the gravitational constant increases with time [24-26]. In the expression of $G$ in equation (33), the scale factor (a) is an increasing function of time. Thus, $G$ would be an increasing function of time only for the negative values of the parameter $l$. Therefore, in the present study we have used only negative values of the parameter $l$.

A quantity $\frac{\dot{G}}{G}$, which can be determined from astrophysical observations, is given by,

$\frac{\dot{G}}{G}=\frac{1}{G} \frac{d G}{d t}=-l \frac{\dot{a}}{a}=-l H=-l\left(\beta+\frac{\alpha}{t}\right)$

Equations (26) and (33) have been used to obtain equation (34).
Using Equation (34) one gets,

$$
\left(\frac{\dot{G}}{G}\right)_{t=t_{0}}=-l H_{0}
$$

where $H_{0}=7.35 \times 10^{-11} y r^{-1}$.

The value of the parameter $l$ should be such, that one must have,

$$
\left|\frac{\dot{G}}{G}\right|_{t=t_{0}}<4 \times 10^{-10} y r^{-1}
$$

This upper limit was shown by Steven Weinberg [27]. These conditions imply that the acceptable values of $l$ must lie in the range of $-5.44<l<0$.

\subsection{Determination of Density Parameters}

Dark energy and matter (dark + baryonic) are regarded as the major constituents of the matter dominated present era of the universe $[8,28]$. Therefore we have, $\rho=\rho_{m}+\rho_{d}$ where $\rho_{m}$ and $\rho_{d}$ denote the densities of matter and dark energy respectively.

The total pressure $(P)$ of the entire matter-energy content of the universe is exerted by dark energy because, the whole matter content (dark + baryonic) is regarded as pressureless dust $[15,29]$. Thus we can write,

$$
P=\gamma \rho=\gamma_{d} \rho_{d}
$$

Here, $\gamma_{d}$ and $\rho_{d}$ are respectively the EoS parameter and density for dark energy. Therefore,

$$
\gamma_{d}=\frac{\gamma \rho}{\rho_{d}}
$$

Using equation (37), the density parameter for dark energy is given by,

$$
\Omega_{d}=\frac{\rho_{d}}{\rho}=\frac{\gamma}{\gamma_{d}}
$$


The density parameter for matter (dark + baryonic) can therefore be expressed as,

$$
\Omega_{m}=\frac{\rho_{m}}{\rho}=\frac{\rho-\rho_{d}}{\rho}=1-\frac{\gamma}{\gamma_{d}}
$$

Considering the fact that the main constituents of the present matter-dominated universe are matter (dark + baryonic) and dark energy, it would be very natural to write,

$$
\Omega_{m}+\Omega_{d}=1
$$

To show the time-dependence of $\Omega_{d}$ and $\Omega_{m}$, we have used an empirically chosen function $f(t)$, which increases with time $(t)$ from zero to one as $t$ changes from zero to infinity. The constants connected to this function can be determined from astrophysical observations. This function is,

$$
f(t)=e^{-\frac{A}{t^{B}}}
$$

Here $A$ and $B$ are constants with $A>0, B>0$. As $t$ increases from zero to infinity, its value remains confined in the interval between zero and unity.

From various studies, it is known that the dark energy content of the universe increase with time, at the cost of matter, due to some interaction between them that causes inter-conversion [20, 21, 29]. Therefore, using the function represented by equation (41), the density parameter for dark energy can be empirically expressed as,

$$
\Omega_{d}=f(t)=e^{-\frac{A}{t^{B}}}
$$

Using equations (40) and (42), the density parameter for matter can be expressed as,

$$
\Omega_{m}=1-\Omega_{d}=1-f(t)=1-e^{-\frac{A}{t^{B}}}
$$

One needs to find the values of the constants $A$ and $B$ from some astrophysical observations.

These observations are listed below:

(1) In the evolution of density parameters of the universe, there was a time in the recent past when $\Omega_{d}=\Omega_{m}=0.5$ and, the corresponding redshift $(z)$ value was lying somewhere in the range of $0<z<$ 1 , as obtained from some recent studies $[21,30]$ in this regard.

Let us define a constant $r$, such that, at $t=r t_{0}$, $\Omega_{d}=\Omega_{m}=\frac{1}{2}$ where $r$ is a fraction.

According to a recent study, the transition of the universe from a phase of decelerated expansion to its present state of accelerated expansion took place in the past at $z=0.6818$ which was around $7.2371 \times$ $10^{9}$ years ago [8], i.e., when the age of the universe was nearly half its present age.

(2) The value of $\Omega_{d 0}$ is close to 0.7 according to several astrophysical observations where $\Omega_{d 0}=$ $\Omega_{d}\left(t=t_{0}\right) \quad$ and $\quad \Omega_{m 0}=\Omega_{m}\left(t=t_{0}\right)$. Therefore, $\Omega_{m 0}$ is approximately 0.3 , as per equation (40) [8, 28].

\section{RESULTS AND DISCUSSION}

Calculation of the constants $A$ and $B$ from the above observations

Using equation (42) and the first one of the above observations we write,

$$
\operatorname{Exp}\left[-A /\left(r t_{0}\right)^{B}\right]=\frac{1}{2}
$$

Using equation (42) and the second one of the above observations we write,

$$
\operatorname{Exp}\left[-A / t_{0}{ }^{B}\right]=\Omega_{d 0}=0.7(\text { approx })
$$


Using equations (44) and (45), one gets the following expressions for the constant parameters $A$ and $B$.

$$
\begin{aligned}
& A=-t_{0}^{B} \ln \left(\Omega_{d 0}\right) \approx-t_{0}^{B} \ln (0.7) \\
& B=\frac{\ln \left[\ln \left(\Omega_{d 0}\right) / \ln (0.5)\right]}{\ln (r)} \approx \frac{\ln [\ln (0.7) / \ln (0.5)]}{\ln (r)}
\end{aligned}
$$

To calculate the value of $A$ from equation (46), one needs to determine the value of $B$ first, from equation (47). For $r=0.6$ one gets $A=3.1811 \times 10^{22}$ and $B=1.3007$ and, for $r=0.5$ one gets $A=$ $2.9232 \times 10^{16}$ and $B=0.9586$ from equations (46) and (47). Thus, for any value of $r$, one can determine these constants, $A \& B$, from equations (46) and (47) respectively. Using the values of $A$ and $B$ in equations (42) and (43), one gets the time variation of $\Omega_{d}$ and $\Omega_{m}$.

Time evolution of the densities of matter and dark energy $\left(\rho_{m}, \rho_{d}\right)$ can be obtained from the equations (42) and (43). Their expressions are given below.

$$
\begin{array}{r}
\rho_{m}=\rho \Omega_{m}=\rho\left(1-e^{-\frac{A}{t^{B}}}\right) \\
\rho_{d}=\rho \Omega_{d}=\rho e^{-\frac{A}{t^{B}}}
\end{array}
$$

In equations (48) and (49), the density $(\rho)$ has to be taken from equation (22).

Combining equation (37) with (49), one gets the following expression for the EoS parameter for dark energy.

$$
\gamma_{d}=\frac{\gamma \rho}{\rho_{d}}=\frac{\gamma}{\Omega_{d}}=\gamma e^{\frac{A}{t^{B}}}
$$

Substituting for $\gamma$, in equation (50), from equation (23), one obtains,

$$
\gamma_{d}=-\left(\frac{2 a_{2}+a_{3}+\frac{\omega}{2} \phi_{1}^{2}+2 a_{1} \phi_{1}+\phi_{2}}{3 a_{3}+3 a_{1} \phi_{1}-\frac{\omega}{2} \phi_{1}^{2}}\right) e^{\frac{A}{t^{B}}}
$$

Taking $\gamma_{0}=-0.75$ from Figure 6 , we get $\gamma_{d 0}=$ $\gamma_{0} \operatorname{Exp}\left[A / t_{0}{ }^{B}\right]=-1.07$. This value of the equation of state $(\mathrm{EoS})$ parameter for dark energy, at the present epoch $\left(t=t_{0}\right)$, is in agreement with other studies on cosmology [21].

Using the fact that $\Omega_{d}=\Omega_{m}$ at the time of $t=r t_{0}$, we get the following expression for the parameter $r$, in terms of the constants $A$ and $B$.

$$
r=\frac{1}{t_{0}}\left(\frac{A}{\ln 2}\right)^{1 / B}
$$

Using equation (52), the value of $r$ can be calculated if one can obtain the values of $A$ and $B$ from the results of other studies on cosmology. Using this value of $r$, one can determine the $\operatorname{redshift}(z)$ value at which the universe had $\Omega_{d}=\Omega_{m}$. This can be done by putting $t=r t_{0}$ in the expression for $z$, represented by equation (32).

\section{FIGURES}

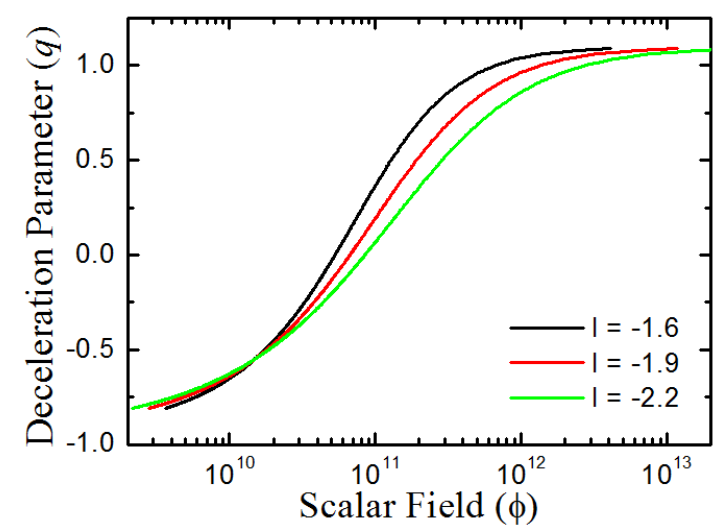

Figure 1: Plots of deceleration parameter versus scalar field, for three values of the parameter $l$. 


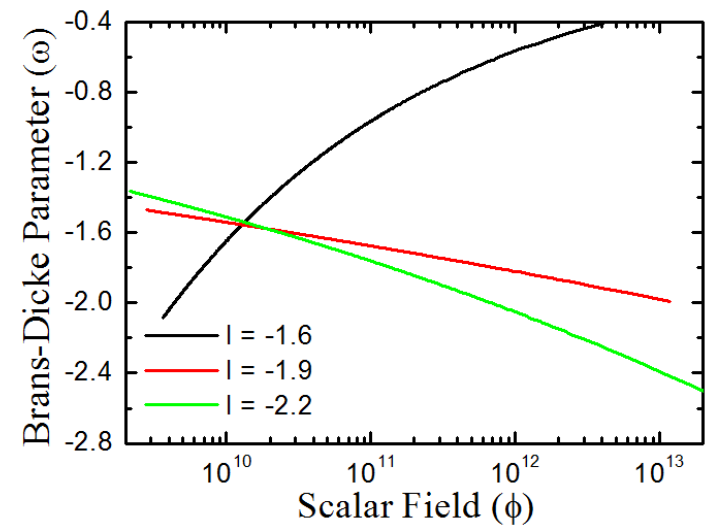

Figure 2: Plots of Brans-Dicke Parameter versus scalar field, for three values of the parameter $l$.

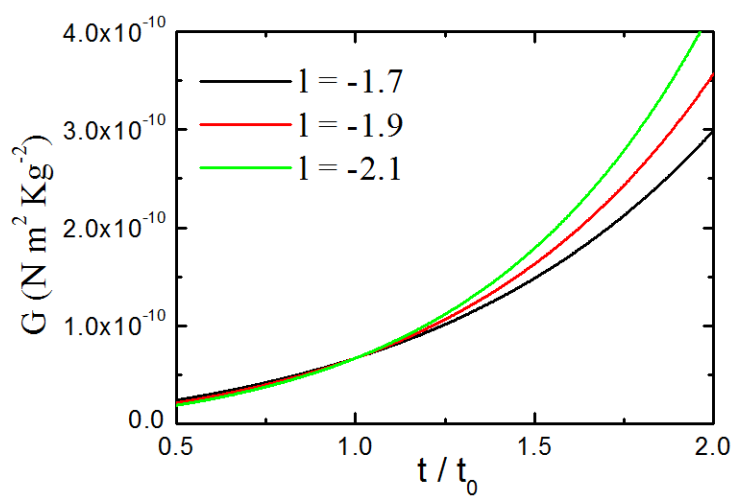

Figure 3: Plots of Gravitational Constant versus time, for three values of the parameter $l$.

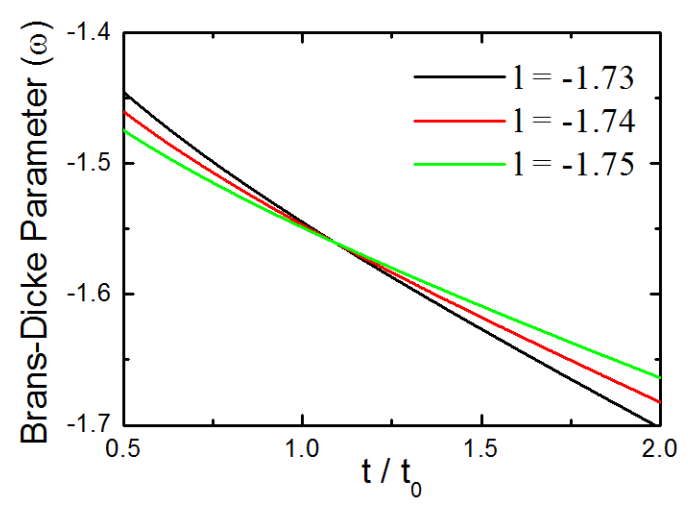

Figure 4: Plots of Brans-Dicke Parameter versus time, for three values of the parameter $l$.

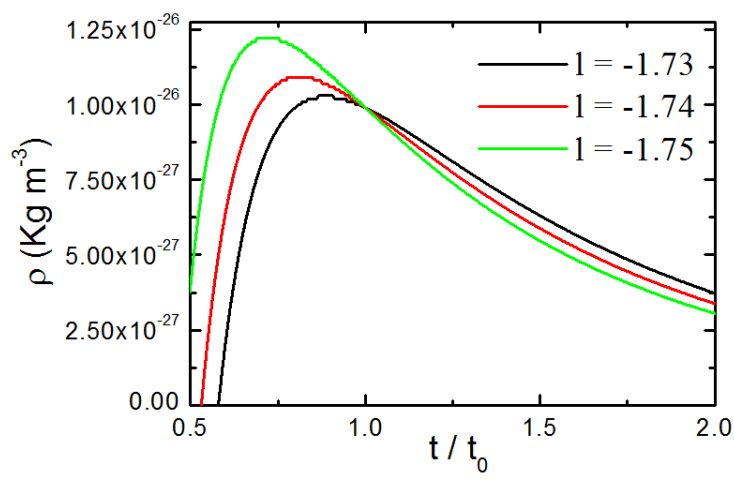

Figure 5: Plots of energy density versus time, for three values of the parameter $l$.

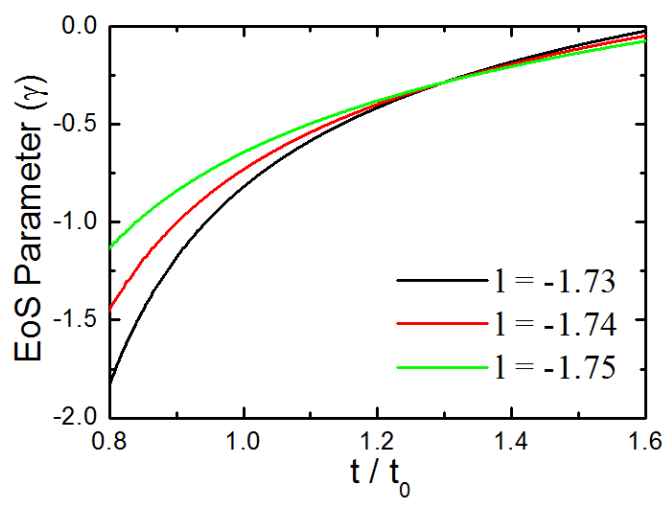

Figure 6: Plots of Equation of State (EoS) Parameter versus time, for three values of the parameter $l$.

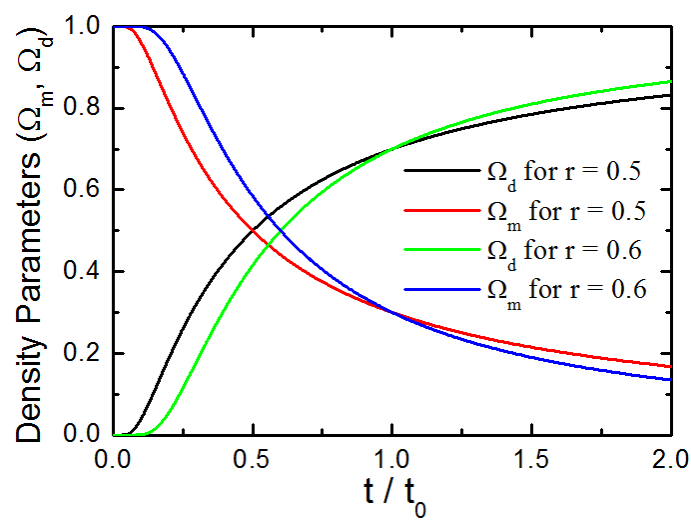

Figure 7: Plots of $\Omega_{m} \& \Omega_{d}$ versus time, for two values of the parameter $r$. 


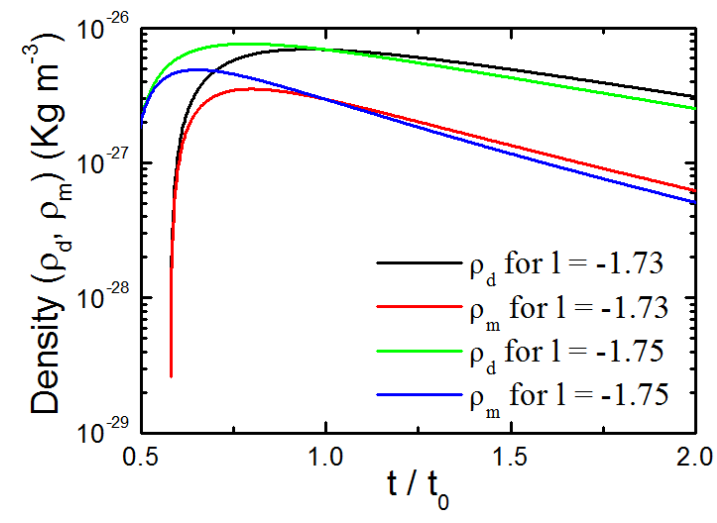

Figure 8: Plots of $\rho_{d} \& \rho_{m}$ versus time, for two values of the parameter $l$. Here, $r=0.5$.

\section{Graphical Depiction of Theoretical Findings}

Figure 1 shows the variation of the deceleration parameter $(q)$ as a function of the scalar field $(\phi)$, for three values of the parameter $l$. Due to the negative values of $l$, the scalar field itself decreases with time, as per equation (14). The deceleration parameter decreases, from positive to negative values, with the decrease of the scalar field. Its rate of decrease is slower for more negative values of the parameter $l$.

Figure 2 shows the variation of the Brans-Dicke Parameter $(\omega)$ as a function of the scalar field $(\phi)$, for three values of the parameter $l$. Due to the negative values of $l$, the scalar field itself decreases with time, as per equation (14). As it decreases, the $\mathrm{BD}$ parameter either decreases or increases with time, depending upon the value of the parameter $l$. For more negative values of this parameter, it increases.

Figure 3 shows the variation of the gravitational constant $(G)$ as a function of time, for three values of the parameter $l$. $G$ is the reciprocal of $\phi$. Due to the negative values of $l, \phi$ decreases with time, as per equation (14). Therefore $G$ increases with time. The rate of increase is faster for more negative values of $l$. This behaviour is consistent with some other studies on cosmology [24-26].
Figure 4 shows the variation of Brans-Dicke parameter $(\omega)$ as a function of time, for three values of the parameter $l$. This parameter becomes more negative with time. Its rate of change is faster for less negative values of the parameter $l$.

Figure 5 shows the variation of matter-energy density $(\rho)$ as a function of time, for three values of the parameter $l$. This is the density of the total content of all constituents of the universe. It initially rises to a peak and then goes on decreasing with time. This attainment of the peak value and the subsequent decrease is faster for more negative values of the parameter 1. This result is consistent with the findings of some other studies [31].

Figure 6 shows the variation of equation of state (EoS) parameter $(\gamma)$ as a function of time, for three values of the parameter $l$. This parameter becomes less negative with time. Its rate of change gradually decreases with time. Less negative values of $l$ cause a faster change. This result is consistent with the findings of some other studies [32].

Figure 7 shows the variation of the cosmological density parameters $(\Omega)$, for both matter and dark energy, as functions of time, for two values of the parameter $r$. The density parameter for matter decreases with time and density parameter for dark energy increases with time. Their sum remains approximately unity at any instant, since they are known to be the main constituents of the present universe. Larger values of the parameter $r$ cause a faster change of the values of density parameters $\left(\Omega_{m}, \Omega_{d}\right)$ with time. Equations (42), (43), (46) and (47) have been used for these plots.

Figure 8 shows the variation of the densities of matter \& dark energy $\left(\rho_{m}\right.$ and $\left.\rho_{d}\right)$ as functions of time, for two values of the parameter $l$. Here, $r=0.5$. 
Both densities are found attain peak values very steeply before beginning to decrease at a much slower pace. More negative values of 1 cause quicker attainment of peak values for both densities. Equations (48) and (49) have been used, in combination with equation (22), for these plots.

\section{CONCLUDING REMARKS}

The present article is based on a study in the framework of Brans-Dicke theory, carried out for a homogeneous and isotropic space-time for a universe with spatial curvature zero. The time dependence of the dimensionless Brans-Dicke parameter $(\omega)$, energy density $(\rho)$ and the EoS parameter $(\gamma)$ have been determined from the field equations, with the help of an empirical expression for the scalar field $(\phi)$ representing its relation with the scale factor. The scale factor has been so chosen that it leads to a signature flip of the deceleration parameter in the course of its time evolution. Without resorting to any self-interaction potential $\left(V_{\phi}\right)$ and cosmological constant $(\Lambda)$, the time variations of the density parameters for matter and dark energy have been evaluated and these findings are very much in agreement with those obtained from other studies. This article provides an empirical formulation of the expressions for the density parameters, which is not based upon any interaction between matter and dark energy. This formulation may be used for other theoretical studies in future. To improve the model for the determination of the density parameters, one may choose a different functional form for the function $f(t)$, instead of that given by equation (41). A more accurate evaluation of the constants, $A$ and $B$, is possible by using more authentic information (based on astrophysical observations) regarding the value of $z$ or $t$ corresponding to the time when the universe had $\Omega_{m}=\Omega_{d}$. As per equation (33), the value of parameter $l$ governs the time variation of the gravitational constant $(G)$. The same parameter controls the nature of time variations of the cosmological quantities ( $\omega, \rho$ and $\gamma)$ discussed in the present article. This fact indicates that the dependence of the scalar field $\phi(\equiv 1 / G)$ upon the scale factor (a) (as given by eqn. 14) has a very significant role in governing cosmic expansion. The dependence of $\omega$ upon $\phi$ (as per the ansatz of eqn. $15)$ is determined by $\omega_{0}$ and $m$, which are all dependent upon the parameter $l$. On the basis of these observations, one may undertake a project in future to improve this model by choosing a relation, between $\phi$ and $a$, which would be different from the ansatz expressed by equation (14).

\section{ACKNOWLEDGEMENT}

The authors of this article are immensely thankful to all academicians and researchers whose works have inspired them to carry out the present study. The theoretical derivations and the calculations for the present article were undertaken during a dissertation project in the final year of the M.Sc. course in Physics, under the supervision of Dr. S. Roy, an author of this article. He expresses his sincere thanks to his colleagues in this regard.

\section{REFERENCES}

[1]. A. G. Riess et al., 1998. Observational Evidence from Supernovae for an Accelerating Universe and a Cosmological Constant, Astron. J., 116: 1009-1038.

[2]. A. G. Riess et al., 2001. The farthest known supernova: support for an accelerating universe and a glimpse of the epoch of deceleration, Astrophys. J., 560(1): 49-71.

[3]. C. L. Bennett et al., 2003. First Year Wilkinson Microwave Anisotropy Probe (WMAP) 
Observations: Preliminary Maps and Basic Results, Astrophys. J. Suppl., 148: 1-27.

[4]. E. Komatsu et al., 2011. Seven-Year Wilkinson Microwave Anisotropy Probe (WMAP) Observations: Cosmological Interpretation, Astrophys. J. Suppl., 192(18): 1-47.

[5]. W. J. Percival et al., 2010. Baryon Acoustic Oscillations in the Sloan Digital Sky Survey Data Release 7 Galaxy Sample, Mon. Not. Roy. Astron. Soc., 401: 2148-2168.

[6]. T. Padmanabhan and T. R. Choudhury, 2003. A theoretician's analysis of the supernova data and the limitations in determining the nature of dark energy, Mon. Not. R. Astron. Soc., 344: 823-834.

[7]. T. R. Choudhury and T. Padmanabhan, 2005. Cosmological parameters from supernova observations: A critical comparison of three data sets, Astronomy and Astrophysics, 429: 807-818.

[8]. G. K. Goswami, 2017. Cosmological parameters for spatially flat dust filled Universe in BransDicke theory, Research in Astronomy and Astrophysics (RAA), 17(3): 1-12.

[9]. C. Brans and R. H. Dicke, 1961. Mach's Principle and a Relativistic Theory of Gravitation, Phys. Rev., 124: 925-935.

[10]. B. K. Sahoo and L. P. Singh, 2002. Time Dependence of Brans-Dicke Parameter $\omega$ for an Expanding Universe, Mod. Phys. Lett. A, 17(36): 2409-2415.

[11]. A. D. Linde, 1990. Extended Chaotic Inflation and Spatial Variations of the Gravitational Constant, Phys. Lett. B, 238: 160-165.

[12]. P. G. Bergmann, 1968. Comments on the scalartensor theory, Int. J. Theor. Phys., 1(1): 25-36.

[13]. R. V. Wagoner, 1970. Scalar-Tensor Theory and Gravitational Waves, Phys. Rev. D, 1(12): 3209-3216.
[14]. K. Nordtvedt Jr., 1970. Post-Newtonian Metric for a General Class of Scalar-Tensor Gravitational Theories and Observational Consequences, Astrophys. J., 161: 1059-1067.

[15]. N. Banerjee and K. Ganguly, 2009. Generalised scalar-tensor theory and the cosmic acceleration, Int. J. Mod. Phys. D, 18: 445-451.

[16]. W. Chakraborty and U. Debnath, 2009. Role of Brans-Dicke Theory with or without selfinteracting potential in cosmic acceleration, Int. J. Theor. Phys., 48(1): 232-247.

[17]. M. Jamil and D. Momeni, 2011. Evolution of the Brans-Dicke Parameter in Generalized Chameleon Cosmology, Chin. Phys. Lett., 28(9): 099801: 1-4.

[18]. J. Satish and R. Venkateswarlu, 2014. Behaviour of Brans-Dicke parameter in generalised chameleon cosmology with Kantowski-Sachs spacetime, Eur. Phys. J. Plus, 129(275): 1-8.

[19]. A. Chand, R. K. Mishra and A. Pradhan, 2016. FRW cosmological models in Brans-Dicke theory of gravity with variable $\mathrm{q}$ and dynamical L-term, Astrophys Space Sci., 361(81): 1-12.

[20]. S. Roy, 2019. Time evolution of the matter content of the expanding universe in the framework of Brans-Dicke gravity, Research in Astronomy and Astrophysics, 19(4): 61-74.

[21]. S. Das and A. Al Mamon, 2014. An Interacting Model of Dark Energy in Brans-Dicke Theory, Astrophys Space Sci., 351(2): 651-660.

[22]. S. Das and N. Banerjee, 2006. An interacting scalar field and the recent cosmic acceleration, Gen. Relativ. Gravit., 38: 785-794.

[23]. S. Roy, S. Chattopadhyay and A. Pasqua, 2013. A study on the dependence of the dimensionless Brans-Dicke parameter on the scalar field and their time dependence, Eur. Phys. J. Plus, 128(147): 1-16. 
[24]. A. Pradhan, B. Saha and V. Rikhvitsky, 2015. Bianchi type-I transit cosmological models with time dependent gravitational and cosmological constants - re-examined, Indian Journal of Physics, 89: 503-513.

[25]. B. Saha, V. Rikhvitsky and A. Pradhan, 2015. Bianchi type-1 cosmological models with time dependent gravitational and cosmological constants: An alternative approach, Rom. Journ. Phys., 60(1-2): 3-14.

[26]. U. Mukhopadhyay, I. Chakraborty, S. Ray and A. A. Usmani, 2016. A Dark Energy Model in Kaluza-Klein Cosmology, Int. J. Theor. Phys., 55(1): 388-395.

[27]. S. Weinberg, 1972. Gravitation and Cosmology: Principles and Applications of the General Theory of Relativity, John Wiley \& Sons, Chapter- 16, pp. 630.

[28]. P. B. Pal, 2000. Determination of cosmological parameters: An introduction for non-specialists, Pramana, 54(1): 79-91.

[29]. H. Farajollahi and N. Mohamadi, 2010. Generalized Brans-Dicke cosmology in the presence of matter and dark energy, Int. J. Theor. Phys., 49: 72-78.

[30]. B. C. Paul, P. Thakur and S. Ghose, 2010. Constraints on Exotic Matter for An Emergent Universe, Mon. Not. Roy. Astron. Soc., 407(1): 415-419.

[31]. A. Pradhan, H. Amirhashchi and B. Saha, 2011. Bianchi Type-I Anisotropic Dark Energy Models with Constant Deceleration Parameter, Int. J. Theor. Phys., 50(9): 2923-2938.

[32]. A. K. Yadav, F. Rahaman and S. Ray, 2011. Dark Energy Models with Variable Equation of State Parameter, Int. J. Theor. Phys., 50(3): 871881.

\section{Cite this article as:}

Sudipto Roy, Avik Ghosh, Adrika Dasgupta, " Time Dependence of Cosmological Parameters in the Framework of Brans-Dicke Theory", International Journal of Scientific Research in Science and Technology(IJSRST), Print ISSN : 2395-6011, Online ISSN : 2395-602X, Volume 6, Issue 5, pp.242-254, September-October-2019. Available at doi :

https://doi.org/10.32628/IJSRST196536

Journal URL : http://ijsrst.com/IJSRST196536 